\title{
Synthesis of (-)- $\Delta^{9}$-trans-Tetrahydrocannabinol - Stereocontrol via Mo- catalyzed Asymmetric Allylic Alkylation Reaction
}

\section{Barry M. Trost* and Kalindi Dogra}

\section{Supporting Information}

$\underline{\text { Table of Contents }}$

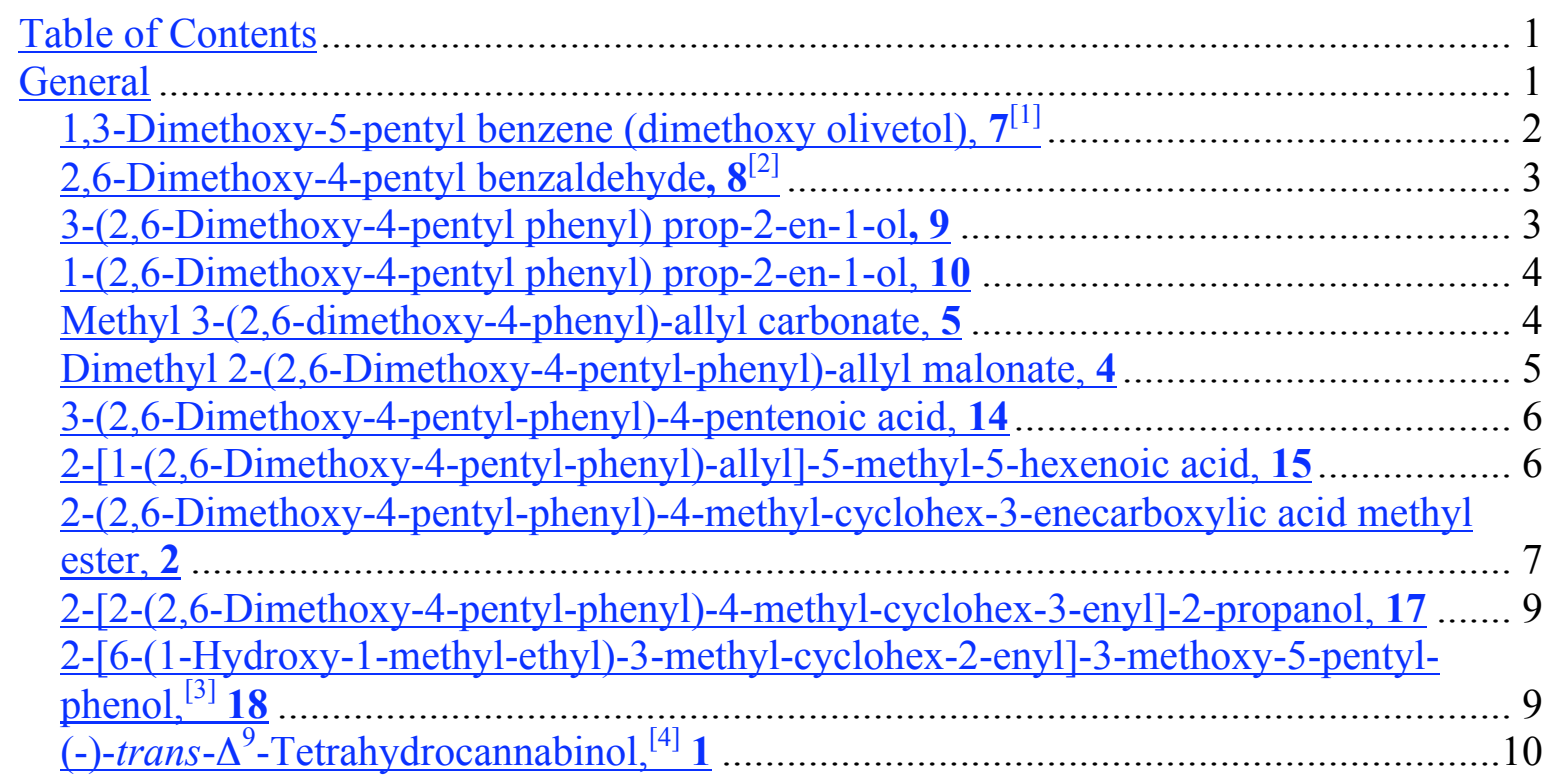

\section{$\underline{\text { General }}$}

All reactions were conducted under a positive pressure of dry argon or nitrogen within glassware that had been oven dried prior to use unless otherwise noted. Anhydrous solutions of reaction mixtures were transferred via an oven dried syringe or cannula. All solvents were distilled from appropriate drying agents prior to use. Diisopropylamine, benzene, acetonitrile, methylene chloride, pyridine and triethylamine were distilled from calcium hydride. Ether and tetrahydrofuran were distilled from sodium benzophenone ketyl. Toluene was distilled from molten sodium. Acetone was distilled from barium oxide. Methanol and ethanol were distilled from magnesium methoxide and ethoxide respectively. Acetonitrile, methylene chloride, toluene and diethyl ether were some times purified by drying column system. Reagents were obtained from Aldrich, Farchan, Fluka, Strem, TCI, or Lancaster and used as obtained unless otherwise noted.

Analytical thin-layer chromatography was performed with $0.2 \mathrm{~mm}$ coated commercial silica gel plates (E. Merck, DC-Plasrikfolien, kieselgel $60 \mathrm{~F}_{254}$ ). Flash Chromatography was 
performed with EM Science silica gel (0.040-0.063 $\mu \mathrm{m}$ grade). Melting points were obtained on a Thomas-Hoover apparatus in open capillary tubes and are uncorrected. Kugelrohr distillations were performed on a Büchi GKR-50 glass tube oven.

Proton nuclear magnetic resonance $\left({ }^{1} \mathrm{H}-\mathrm{NMR}\right)$ data were acquired at $300 \mathrm{MHz}$ on a Varian GEM-300 and at $500 \mathrm{MHz}$ on a Varian GEM-500 spectrometer. Chemical shifts are reported in delta $(\delta)$ units, in parts per million $(\mathrm{ppm})$ downfield from tetramethylsilane, of in ppm relative to the singlet at $7.26 \mathrm{ppm}$ from chloroform- $d$. Splitting patterns are designated as s, singlet; d, doublet,; t, triplet; q, quartet; p, pentet, m, multiplet, br, broad. Carbon-13 nuclear magnetic resonance $\left({ }^{13} \mathrm{C}-\mathrm{NMR}\right)$ data were acquired at $75 \mathrm{MHz}$ on a Varian GEM-300 and at $125 \mathrm{MHz}$ on a Varian GEM-500 spectrometer. Chemical shifts are reported in ppm relative to the center line of a triplet at $77.0 \mathrm{ppm}$ for choroform- $d$ and the center line of a septet at $39.5 \mathrm{ppm}$ in dimethylsulfoxide- $d_{6}$.

Infrared (IR) data were recorded as films on sodium chloride plates or a potassium bromide $(\mathrm{KBr})$ pellets on a Perkin-Elmer Paragon 500 FT-IR spectrometers. Absorbance frequencies are reported in reciprocal centimeters $\left(\mathrm{cm}^{-1}\right)$. High resolution mass spectral data (HRMS) were obtained from Mass Spectrometry Resource, School of Pharmacy, University of California-San Francisco, on a Kratos MS9 spectrometer at an ionizing current of $98 \mathrm{~mA}$ and an ionizing voltage of $70 \mathrm{eV}$. Elemental analyses (Anal.) were performed by M.-H.-W. Laboratories of Pheonix, AZ.

1,3-Dimethoxy-5-pentyl benzene (dimethoxy olivetol), $7^{[1]}$<smiles>COc1cc([18OH])cc(OC)c1</smiles>

To olivetol (3 g, $16.6 \mathrm{mmol})$ and potassium carbonate $(6.9 \mathrm{~g}, 49.9 \mathrm{mmol})$ in $30 \mathrm{ml}$ dry acetone at room temperature is added methylsulfate $(6.3 \mathrm{~g}, 49.9 \mathrm{mmol})$ and the resulting mixture refluxed for $4 \mathrm{~h}$, cooled, filtered and residue washed with ether. The filtrate was washed with $1 \mathrm{M} \mathrm{HCl}(10 \mathrm{ml})$ and water $(2 \times 10 \mathrm{ml})$, dried over $\mathrm{MgSO}_{4}$, concentrated and chromatographed (5\% ethyl acetate in petroleum ether) to yield 3.38g (98\%). ${ }^{1} \mathrm{H}$ NMR $\left(300 \mathrm{MHz}, \mathrm{CDCl}_{3}\right) \delta$ : $6.344(\mathrm{~d}, J=2.198 \mathrm{~Hz}, 1 \mathrm{H}), 6.297(\mathrm{~d}, J=2.198 \mathrm{~Hz}, 1 \mathrm{H}) 3.779(\mathrm{~s}, 6 \mathrm{H}), 2.541(\mathrm{t}, J=7.569$ $\mathrm{Hz}, 2 \mathrm{H}), 1.56-1.62(\mathrm{~m}, 2 \mathrm{H}), 1.28-1.4(\mathrm{~m}, 4 \mathrm{H}), 0.89$ (t, $J=6.837 \mathrm{~Hz}, 3 \mathrm{H})$. 
2,6-Dimethoxy-4-pentyl benzaldehyde, $\mathbf{8}^{[2]}$<smiles>COc1cc([12CH2])cc(OC)c1C=O</smiles>

To 7 (5 g, $24 \mathrm{mmol})$ in 50ml THF at room temperature was added BuLi (1.36M in hexane, $19.41 \mathrm{ml}, 26.4 \mathrm{mmol}$ ) and the resulting was refluxed under nitrogen for $2.5 \mathrm{~h}$, cooled to room temperature and dimethyl formamide $(1.93 \mathrm{~g}, 26.4 \mathrm{mmol})$ added to it. The resulting was refluxed for further $3 \mathrm{~h}$, cooled to room temperature, quenched with water and saturated ammonium chloride solution. The aqueous layer was extracted with ether $(2 \times 100 \mathrm{ml})$. Organic layer was dried over $\mathrm{MgSO}_{4}$, concentrated and chromatographed (5-10\% ethyl acetate in petroleum ether) to yield 4.71g (83\%). ${ }^{1} \mathrm{H}$ NMR $\left(300 \mathrm{MHz}, \mathrm{CDCl}_{3}\right) \delta: 10.45(\mathrm{~s}$, $1 \mathrm{H}), 6.387(\mathrm{~s}, 2 \mathrm{H}), 3.888(\mathrm{~s}, 6 \mathrm{H}), 2.6(\mathrm{t}, J=7.569 \mathrm{~Hz}, 2 \mathrm{H}), 1.56-1.7(\mathrm{~m}, 2 \mathrm{H}), 1.28-1.42(\mathrm{~m}$, $4 \mathrm{H}), 0.91(\mathrm{t}, J=6.592 \mathrm{~Hz}, 3 \mathrm{H})$.

3-(2,6-Dimethoxy-4-pentyl phenyl) prop-2-en-1-ol, 9<smiles>COc1cc([12CH2])cc(OC)c1/C=C/CO</smiles>

To a suspension of sodium hydride (60\% in oil; $0.818 \mathrm{~g}, 20.5 \mathrm{mmol})$ in THF (50ml) was added dropwise triethylphosphonoacetate $(4.08 \mathrm{ml}, 20.5 \mathrm{mmol})$. The clear solution was then cooled to $-35^{\circ} \mathrm{C}$ and a solution of the 2,6-Dimethoxy-4-pentyl benzaldehyde (4.395 g, 18.59 $\mathrm{mmol}$ ) in $10 \mathrm{ml}$ THF was added drop wise. The mixture was then allowed to warm to room temperature, and stirred at same for $1 \mathrm{hr}$ till all starting material was seen to disappear by TLC. It was then quenched by addition of saturated aqueous ammonium chloride $(50 \mathrm{ml})$. The layers were separated and the aqueous layer was extracted with ether $(3 \times 100 \mathrm{ml})$. The combined organic layers were dried over $\mathrm{MgSO}_{4}$ and concentrated down to $60 \mathrm{ml}$. To the cooled $\left(-10^{\circ} \mathrm{C}\right)$ solution of the above formed $\alpha, \beta$-unsaturated ethyl ester was added drop wise $46.5 \mathrm{ml}$ diisobutylaluminium hydride ( $46.5 \mathrm{mmol}$; $1 \mathrm{M}$ in hexane) and the mixture was stirred $30 \mathrm{~min}$ at $0^{\circ} \mathrm{C}$ till TLC showed no starting material. Saturated aqueous ammonium chloride $(10 \mathrm{ml})$ was then carefully added followed by $\mathrm{MgSO}_{4}$ and the resulting slurry stirred for half hour. The suspension was filtered and washed with ether $(300 \mathrm{ml})$ and $\mathrm{CH}_{2} \mathrm{Cl}_{2}(300 \mathrm{ml})$. Evaporation of the solvent gave the crude product, which was purified by flash chromatography using $10 \%$ ethyl acetate- petroleum ether to yield $4.8 \mathrm{~g}(97 \%)$ of the desired 
alcohol as a pale yellow solid. $\mathrm{Rf}=0.386(10 \%$ ethyl acetate in petroleum ether). IR (thin film): $3377.7,2931.2,2856.7,1605.9,1573.2,1456.4,1239.6,1127.0,978.2 \mathrm{~cm}^{-1}$. ${ }^{1} \mathrm{H}$ NMR $\left(500 \mathrm{MHz}, \mathrm{CDCl}_{3}\right) \delta: 6.861(\mathrm{~d}, J=16.113 \mathrm{~Hz}, 1 \mathrm{H}), 6.744(\mathrm{dt}, J=16.113,6.104 \mathrm{~Hz}, 1 \mathrm{H})$, $6.382(\mathrm{~s}, 2 \mathrm{H}), 4.304$ (dd, $J=6.104,0.977 \mathrm{~Hz}, 2 \mathrm{H}), 3.839$ (s, 6H), 2.569 (t, $J=7.569 \mathrm{~Hz}, 2 \mathrm{H})$, $1.58-1.65(\mathrm{~m}, 2 \mathrm{H}), 1.3-1.38(\mathrm{~m}, 4 \mathrm{H}), 0.901(\mathrm{t}, J=6.836 \mathrm{~Hz}, 3 \mathrm{H}) .{ }^{13} \mathrm{C}$ NMR $(125 \mathrm{MHz}$, $\left.\mathrm{CDCl}_{3}\right) \delta: 158.26,143.8,131.45,121.07,111.15,104.01,65.62,55.6,36.61,31.54,31.03$, 22.53, 14.03. Elemental analysis calculated for $\mathrm{C}_{16} \mathrm{H}_{24} \mathrm{O}_{3} \mathrm{C}, 72.69$; H, 9.15; found: $\mathrm{C}, 72.88$; H, 9.16.

1-(2,6-Dimethoxy-4-pentyl phenyl) prop-2-en-1-ol, 10<smiles>C=CC(O)c1c(OC)cc([15CH2])cc1OC</smiles>

To $7(0.12 \mathrm{~g}, 0.576 \mathrm{mmol})$ in $5 \mathrm{ml} \mathrm{THF}$ at room temperature was added BuLi $(2.5 \mathrm{M}$ in hexane, $0.253 \mathrm{ml}, 0.634 \mathrm{mmol}$ ) and the resulting was refluxed under nitrogen for $2 \mathrm{~h}$, cooled to $-78^{\circ} \mathrm{C}$ and freshly distilled acrolein $(64.6 \mathrm{mg}, 1.152 \mathrm{mmol})$ added to it. The resulting was stirred at room temperature for $2 \mathrm{~h}$, quenched with water and saturated ammonium chloride solution. The aqueous layer was extracted with ether $(2 \times 10 \mathrm{ml})$. Organic layer was dried over $\mathrm{MgSO}_{4}$, concentrated and chromatographed (5-10\% ethyl acetate in petroleum ether) to yield $0.138 \mathrm{~g}(90.7 \%)$ of the alcohol. $\mathrm{Rf}=0.41$ (10\% ethyl acetate in petroleum ether). IR (thin film): $3560.6,2932.7,2857.9,1610.0,1583.1,1457.5,1420.4,1233.9,1121.7 \mathrm{~cm}^{-1} .{ }^{1} \mathrm{H}$

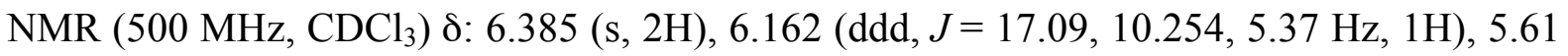
$(\mathrm{OH}, 1 \mathrm{H}), 5-5.22(\mathrm{~m}, 2 \mathrm{H}), 3.929(\mathrm{~d}, J=11.475 \mathrm{~Hz}, 1 \mathrm{H}), 3.82(\mathrm{~s}, 6 \mathrm{H}), 2.559$ (t, $J=7.813 \mathrm{~Hz}$, $2 \mathrm{H}), 1.5-1.7(\mathrm{~m}, 2 \mathrm{H}), 1.25-1.4(\mathrm{~m}, 4 \mathrm{H}), 0.9(\mathrm{t}, J=6.837 \mathrm{~Hz}, 3 \mathrm{H}) .{ }^{13} \mathrm{C} \mathrm{NMR}(75 \mathrm{MHz}$, $\mathrm{CDCl}_{3}$ ) $\delta: 157.42,144.13,140.43,115.66,113.05,104.6,55.72,36.53,31.52,31.06,22.52$, 14.02. Elemental analysis calculated for $\mathrm{C}_{16} \mathrm{H}_{24} \mathrm{O}_{3} \mathrm{C}, 72.69 ; \mathrm{H}, 9.15$; found: $\mathrm{C}, 72.71 ; \mathrm{H}$, 8.92 .

Methyl 3-(2,6-dimethoxy-4-phenyl)-allyl carbonate, 5<smiles>COc1cc([12CH2])cc(OC)c1/C=C/COC(C)=O</smiles> 
To $9(3.75 \mathrm{~g}, 14.18 \mathrm{mmol})$ in $200 \mathrm{ml}$ ether at $-78^{\circ} \mathrm{C}$ is added BuLi $(1.36 \mathrm{M}$ in hexane, $11.5 \mathrm{ml}$, $15.6 \mathrm{mmol})$ with stirring. To the resulting bright red solution methyl chloroformate $(2.68 \mathrm{~g}$, $28.36 \mathrm{mmol}$ ) is added in one shot and the reaction stirred for $10 \mathrm{~min}$. The reaction is then diluted with $100 \mathrm{ml}$ ether and washed twice with ice water. The organic layer is dried over $\mathrm{MgSO}_{4}$ and the solvent removed to give the carbonate as a pale yellow solid in quantitative yield. $\mathrm{Rf}=0.342(5 \%$ ethyl acetate in petroleum ether).

IR (thin film): 2958.5, 2857.1, 1748.1, 1606.3, 1573.6, 1455.7, 1419.8, 1262.2, 1134.0, 942.7 $\mathrm{cm}^{-1} .{ }^{1} \mathrm{H}$ NMR $\left(300 \mathrm{MHz}, \mathrm{CDCl}_{3}\right) \delta: 6.979(\mathrm{~d}, J=16.114 \mathrm{~Hz}, 1 \mathrm{H}), 6.6850$ (dt, $J=16.114$, $6.592 \mathrm{~Hz}, 1 \mathrm{H}), 6.365$ (s, 2H), $4.788(\mathrm{dd}, J=6.592,0.977 \mathrm{~Hz}, 2 \mathrm{H}), 3.833(\mathrm{~s}, 6 \mathrm{H}), 3.788$ (s, $3 \mathrm{H}), 2.564(\mathrm{t}, J=8 \mathrm{~Hz}, 2 \mathrm{H}), 1.56-1.64(\mathrm{~m}, 2 \mathrm{H}), 1.3-1.38(\mathrm{~m}, 4 \mathrm{H}), 0.898$ (t, $J=6.836 \mathrm{~Hz}$, $3 \mathrm{H}) .{ }^{13} \mathrm{C}$ NMR $\left(125 \mathrm{MHz}, \mathrm{CDCl}_{3}\right) \delta: 158.49,155.71,144.35,125.79,124.81,110.58$, $103.89,70.75,55.55,54.58,36.6,31.48,30.69,22.49,13.99$. Elemental analysis calculated for $\mathrm{C}_{18} \mathrm{H}_{26} \mathrm{O}_{5} \mathrm{C}, 67.06 ; \mathrm{H}, 8.13$; found: $\mathrm{C}, 67.0 ; \mathrm{H}, 7.96$.

Dimethyl 2-(2,6-Dimethoxy-4-pentyl-phenyl)-allyl malonate, 4<smiles>C=CC(c1c(OC)cc([13CH3])cc1OC)C(C(C)=O)C(C)=O</smiles>

$\mathrm{Mo}(\mathrm{CO})_{3}\left(\mathrm{C}_{7} \mathrm{H}_{8}\right)(13.6 \mathrm{mg}, 0.05 \mathrm{mmol})$ and ligand $(S, S)-\mathbf{1 1}(24.3 \mathrm{mg}, 0.075 \mathrm{mmol})$ in THF and carbonate 5 (161.2 $\mathrm{mg}, 0.5 \mathrm{mmol})$, dimethyl malonate $(79.3 \mathrm{mg}, 0.6 \mathrm{mmol})$, and $\mathrm{NaH}$ (60\% in oil) (24 mg, $0.6 \mathrm{mmol})$ in THF were reacted $\left(75^{\circ} \mathrm{C}\right.$ oil bath temp.) for $54 \mathrm{hr}$. Work up and flash chromatography (10\% ethyl acetate in petroleum ether) gave $158 \mathrm{mg}(83.6 \%)$ of the branched product. $\mathrm{Rf}=0.38$ (10\% ethyl acetate in petroleum ether), HPLC (OD, flow rate $=1,99: 1$ heptane $/ i$-propanol, 254): 97\%ee, $\mathrm{t}_{\mathrm{r}}=11.74$ (major isomer), $\mathrm{t}_{\mathrm{r}}=15.46$ (minor isomer). $[\alpha]^{\mathrm{D}}=39.2515\left(\mathrm{c}=1.01, \mathrm{CH}_{2} \mathrm{Cl}_{2}\right)$, IR (thin film): 2953.6, 2857.4, 1760.0, 1739.9, 1608.1, 1582.0, 1456.8, 1233.5, $1127.5 \mathrm{~cm}^{-1}$. ${ }^{1} \mathrm{H}$ NMR (500 MHz, $\left.\mathrm{CDCl}_{3}\right) \delta: 6.336(\mathrm{~s}, 2 \mathrm{H})$, $6.116(\mathrm{ddd}, J=17.09,10.254,8.545 \mathrm{~Hz}, 1 \mathrm{H}), 4.95-5.15(\mathrm{~m}, 2 \mathrm{H}), 4.781$ (dd, $J=11.475$, $8.545 \mathrm{~Hz}, 1 \mathrm{H}), 4.461$ (d, $J=11.475 \mathrm{~Hz}, 1 \mathrm{H}), 3.818$ (s, 6H), 3.734 (s, 3H), 3.417 (s, 3H), $2.524(\mathrm{t}, J=8 \mathrm{~Hz}, 2 \mathrm{H}), 1.56-1.64(\mathrm{~m}, 2 \mathrm{H}), 1.26-1.38(\mathrm{~m}, 4 \mathrm{H}), 0.892(\mathrm{t}, J=7.08 \mathrm{~Hz}, 3 \mathrm{H}) .{ }^{13} \mathrm{C}$ NMR (75 MHz, $\left.\mathrm{CDCl}_{3}\right)$ \&: 169.28, 168.52, 158.06,143.47, 136.95,116.06, 113.41, 104.56, $55.8,54.41,52.26,51.95,39.8,36.45,31.56,30.95,22.5,14.01$. Elemental analysis calculated for $\mathrm{C}_{21} \mathrm{H}_{30} \mathrm{O}_{6} \mathrm{C}, 66.65 ; \mathrm{H}, 7.99$; found: $\mathrm{C}, 66.43 ; \mathrm{H}, 8.12$. 
3-(2,6-Dimethoxy-4-pentyl-phenyl)-4-pentenoic acid, 14<smiles>C=C[C@H](CC)c1c(OC)cc([13CH3])cc1OC</smiles>

Alkylation product $4(1.2 \mathrm{~g}, 3.17 \mathrm{mmol})$ from Molybdenum reaction in $1 \mathrm{ml} \mathrm{MeOH}$ and $10 \mathrm{ml}$ of $1 \mathrm{~N}$ aqueous $\mathrm{NaOH}$ was refluxed for $6 \mathrm{hr}$. The resulting solution was cooled to room temperature and acidified. It was extracted with $\mathrm{DCM}$, dried over $\mathrm{MgSO}_{4}$ and concentrated invacuo to give a mixture of malonic acid and the desired product. Heating this mixture at $160^{\circ} \mathrm{C}$ for about an hour gave $0.956 \mathrm{~g}(98.4 \%)$ of the desired acid. $\mathrm{Rf}=0.168(20 \%$ ethyl acetate in petroleum ether). $[\alpha]^{\mathrm{D}}=11.8031\left(\mathrm{c}=1.3, \mathrm{CH}_{2} \mathrm{Cl}_{2}\right)$. IR (thin film): 3078.3, 2932.7, 2677.7, 1705.6, 1582.0, 1455.7, 1418.5, 1233.9, 1123.0, $911.9 \mathrm{~cm}^{-1}$. ${ }^{1} \mathrm{H}$ NMR $(500 \mathrm{MHz}$, $\left.\mathrm{CDCl}_{3}\right) \delta: 6.357(\mathrm{~s}, 2 \mathrm{H}), 6.168(\mathrm{ddd}, J=17.334,10.09,7.324 \mathrm{~Hz}, 1 \mathrm{H}), 4.93-5.08(\mathrm{~m}, 2 \mathrm{H})$, 4.46-4.53 (m, 1H), $3.791(\mathrm{~s}, 6 \mathrm{H}), 3.046(\mathrm{dd}, J=15.625,9.27 \mathrm{~Hz}, 1 \mathrm{H}), 2.74(\mathrm{dd}, J=15.625$, $5.86 \mathrm{~Hz}, 1 \mathrm{H}), 2.542$ (t, $J=7.813 \mathrm{~Hz}, 2 \mathrm{H}), 1.57-1.64$ (m, 2H), 1.29-1.39 (m, 4H), 0.901 (t, $J$ $=7.08 \mathrm{~Hz}, 3 \mathrm{H}) .{ }^{13} \mathrm{C} \mathrm{NMR}\left(125 \mathrm{MHz}, \mathrm{CDCl}_{3}\right) \delta: 178.39,157.93,143.06,139.38,115.77$, $114.32,104.56,55.71,37.72,36.48,35.45,31.65,31.08,22.55,14.05$. Elemental analysis calculated for $\mathrm{C}_{18} \mathrm{H}_{26} \mathrm{O}_{4} \mathrm{C}, 70.56 ; \mathrm{H}, 8.55$; found: C, 70.66; H, 8.7.

2-[1-(2,6-Dimethoxy-4-pentyl-phenyl)-allyl]-5-methyl-5-hexenoic acid, 15

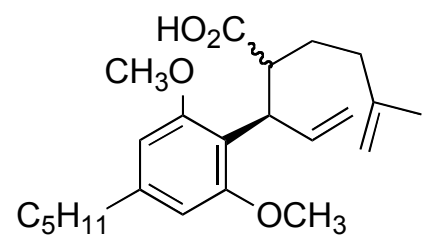

To a $-20^{\circ} \mathrm{C}$ solution of acid $14(2.1 \mathrm{~g}, 6.85 \mathrm{mmol})$ in $5 \mathrm{ml}$ THF was added LDA (prepared from diisopropylamine $(1.66 \mathrm{~g}, 16.45 \mathrm{mmol})$ and $\mathrm{BuLi}(8.45 \mathrm{ml}, 1.95 \mathrm{M}$ in pentane $)$ in $10 \mathrm{ml}$ THF) drop wise with stirring. The solution turns red towards the end. It was then warmed at $40^{\circ} \mathrm{C}$ for half hour and cooled to $-10^{\circ} \mathrm{C}$. To this was added 4-iodo-2-methyl-but-1-ene $(2.02 \mathrm{~g} 10.3 \mathrm{mmol})$ in one shot. The resulting was stirred at $\mathrm{rt}$ for $3 \mathrm{hr}$ in dark, diluted with 50 $\mathrm{ml}$ ether and quenched with $20 \mathrm{ml}$ ice cold $1 \mathrm{~N} \mathrm{HCl}$. The two layers were separated and the aqueous layer extracted with ether $(2 \times 50 \mathrm{ml})$. Combined organic layers were dried over $\mathrm{MgSO}_{4}$, concentrated and the residue columned over silica with $20 \%$ ethyl acetate- petroleum ether to give $84 \%$ of the alkylated product as two diastereomers in a 2.37:1 ratio. Yield: 
major diastereomer $\left(1^{\text {st }}\right.$ running, trans) $1.518 \mathrm{~g}$, minor diastereomer $\left(2^{\text {nd }}\right.$ running, cis) $0.6399 \mathrm{~g}$.<smiles>C=C[C@H](CCC(=C)C)[C@H](C=C)C(=O)O</smiles>

$\mathrm{Rf}=0.35(20 \%$ ethyl acetate in petroleum ether $) \cdot[\alpha]^{\mathrm{D}}=60.739(\mathrm{c}$ $=0.53, \mathrm{CH}_{2} \mathrm{Cl}_{2}$ ). IR (thin film): 3075.8, 2933.3, 2675.9, 1703.4, 1581.4, 1461.1, 1423.0, 1237.4, 1128.1, 911.9, 888.0, 827.1 $\mathrm{cm}^{-1} .{ }^{1} \mathrm{H}$ NMR (500 MHz, $\left.\mathrm{CDCl}_{3}\right) \delta: 6.309$ (s, 2H), $6.176(\mathrm{td}, J=16.968,9.644 \mathrm{~Hz}, 1 \mathrm{H}), 4.97-5.13(\mathrm{~m}, 2 \mathrm{H}), 4.668(\mathrm{~d}, J=14.038 \mathrm{~Hz}, 2 \mathrm{H}), 4.104$ $(\mathrm{t}, J=10.01 \mathrm{~Hz}, 1 \mathrm{H}), 3.744(\mathrm{~s}, 6 \mathrm{H}), 3.179(\mathrm{td}, J=10.865,3.54 \mathrm{~Hz}, 1 \mathrm{H}), 2.509(\mathrm{t}, J=8 \mathrm{~Hz}$, 2H), 1.95- $2.04(\mathrm{~m}, 2 \mathrm{H}), 1.77-1.85(\mathrm{~m}, 1 \mathrm{H}), 1.706(\mathrm{~s}, 3 \mathrm{H}), 1.63-1.71(\mathrm{~m}, 1 \mathrm{H}), 1.54-1.62(\mathrm{~m}$, $2 \mathrm{H}), 1.27-1.38(\mathrm{~m}, 4 \mathrm{H}), 0.898(\mathrm{t}, J=6.958 \mathrm{~Hz}, 3 \mathrm{H}) .{ }^{13} \mathrm{C} \mathrm{NMR}\left(125 \mathrm{MHz}, \mathrm{CDCl}_{3}\right) \delta: 179.44$, 157.92, 145.28, 138.35, 116.27, 115.28, 110.18, 104.8, 55.81, 47.06, 43.08, 36.48, 35.61, 31.7, 31.0, 29.44, 22.55, 22.41, 14.07. Elemental analysis calculated for $\mathrm{C}_{23} \mathrm{H}_{34} \mathrm{O}_{4} \mathrm{C}, 73.76$; H, 9.15; found: C, 73.89; H, 9.46.<smiles>C=CC(CCC(=C)C)C(C=C)c1c(OC)cc([Hg]c2ccccc2)cc1OC</smiles>

$\mathrm{Rf}=0.25$ (20\% ethyl acetate in petroleum ether). IR (thin film): 3075.4, 2933.3, 2668.7, 1704.4, 1581.5, 1455.3, 1419.0, 1235.4, 1128.2, 914.2, 888.5, 823.7 $\mathrm{cm}^{-1}$. ${ }^{1} \mathrm{H}$ NMR $\left(500 \mathrm{MHz}, \mathrm{CDCl}_{3}\right) \delta: 6.354$ (s, 2H), 6.265 (ddd, $J=17.09,10.01,9.033 \mathrm{~Hz}$, 1H), 4.9-5.1 (m, 2H), 4.589 (d, $J=41.748 \mathrm{~Hz}, 2 \mathrm{H}), 4.24$ (t, $J=9.033 \mathrm{~Hz}, 1 \mathrm{H}), 3.794$ (s, 6H), $3.26(\mathrm{td}, J=10.864,3.54 \mathrm{~Hz}, 1 \mathrm{H}), 2.547$ (t, $J=7.81 \mathrm{~Hz}, 2 \mathrm{H}), 1.83-2.18$ (m, 2H), 1.54- 1.66 $(\mathrm{m}, 3 \mathrm{H}), 1.537(\mathrm{~s}, 3 \mathrm{H}), 1.26-1.4(\mathrm{~m}, 5 \mathrm{H}), 0.898(\mathrm{t}, J=7.08 \mathrm{~Hz}, 3 \mathrm{H})$.

${ }^{13} \mathrm{C}$ NMR (125 MHz, $\mathrm{CDCl}_{3}$ ) $\delta: 181.08,158.12,145.07,138.38,115.41,114.12,110.18$, $104.4,55.55,47.48,42.5,36.48,35.16,31.61,31.06,28.26,22.56,22.11,14.07$.

2-(2,6-Dimethoxy-4-pentyl-phenyl)-4-methyl-cyclohex-3-enecarboxylic acid methyl ester, 2<smiles></smiles> 
$\mathrm{Rf}=0.31$ (5\% ethyl acetate in petroleum ether). To a mixture of alkylated acid (1.5 g, 4 $\mathrm{mmol})$ and $\mathrm{K}_{2} \mathrm{CO}_{3}(1.66 \mathrm{~g}, 12 \mathrm{mmol})$ in $30 \mathrm{ml}$ acetone at $\mathrm{rt}$, is added dimethyl sulfate $(1.51 \mathrm{~g}$, $12 \mathrm{mmol}$ ) and the resulting refluxed for $3 \mathrm{hr}$. Excess methyl sulfate is quenched by stirring for an hour with $3 \mathrm{ml}$ methanol. The reaction mixture is cooled, filtered, diluted with ether $(100 \mathrm{ml})$, washed with water $(2 \times 50 \mathrm{ml})$, dried over $\mathrm{MgSO}_{4}$ and concentrated. The concentrated mixture is passed through a plug of silica, and further concentrated to give the methyl ester of the acid in almost quantitative yield. The ester is then dissolved in DCM $(10 \mathrm{ml})$ under nitrogen and added to Grubb's II catalyst (68 $\mathrm{mg}, 0.08 \mathrm{mmol}$ ) via cannula and stirred at $\mathrm{rt}$ overnight. A second portion of catalyst $(34 \mathrm{mg}, 0.04 \mathrm{mmol})$ is then added and the reaction stirred till no more starting material could be seen by TLC $(\sim 16 \mathrm{hr})$. The solvent was evaporated and the residue columned over silica with 5\% ethyl acetate-petroleum ether to yield $1.34 \mathrm{~g}(92.9 \%)$ of the cyclized product. IR (thin film): 2930.1, 2857.5, 1736.1, 1608.0, 1580.9, 1455.0, 1419.8, 1232.0, 1232.0, 1160.0, $1119.5 \mathrm{~cm}^{-1}$. ${ }^{1} \mathrm{H}$ NMR (500 MHz, $\left.\mathrm{CDCl}_{3}\right) \delta$ : 6.342 (s, 2H), 5.152 (s, br, 1H), 4.23 (d, br, $J=8.667 \mathrm{~Hz}, 1 \mathrm{H}), 3.747$ (s, 6H), 3.465 (s, 3H), 3.14- $3.2(\mathrm{~m}, 1 \mathrm{H}), 2.535$ (t, $J=8 \mathrm{~Hz}, 2 \mathrm{H}), 1.82-2.2(\mathrm{~m}, \mathrm{H}), 1.66(\mathrm{~s}, 3 \mathrm{H}), 1.57-1.63(\mathrm{~m}, 2 \mathrm{H})$, 1.26-1.38 (m, 4H), $0.901(\mathrm{t}, J=6.958 \mathrm{~Hz}, 3 \mathrm{H}) .{ }^{13} \mathrm{C} \mathrm{NMR}\left(125 \mathrm{MHz}, \mathrm{CDCl}_{3}\right) \delta: 176.76$, $158.61,142.55,130.94,124.58,117.34,104.91,56.75,51.13,43.34,36.43,34.79,31.65$, $31.03,29.39,27.11,23.35,22.56,14.08$. HRMS calculated for $\mathrm{C}_{22} \mathrm{H}_{32} \mathrm{O}_{4}$ (360.2301), found $=360.2308$.

Equilibrating the cis-ester to the trans-ester
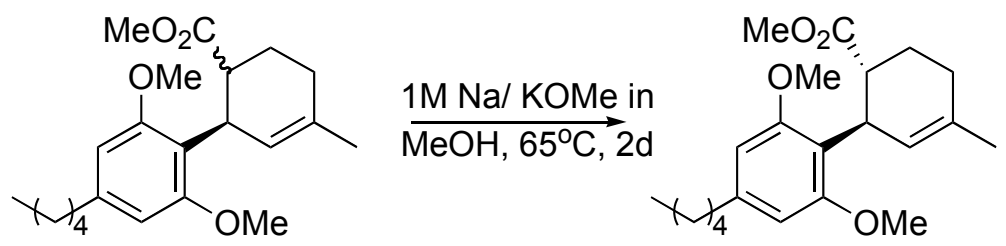

Solution of cis-ester $16(68 \mathrm{mg}, 0.188 \mathrm{mmol})$ in $1 \mathrm{ml}$ freshly prepared $1 \mathrm{M} \mathrm{NaOMe}$ in methanol was stirred for 3 days at $65^{\circ} \mathrm{C}$. The reaction mixture was neutralized with dilute $\mathrm{HCl}$, taken up in $25 \mathrm{ml}$ ether and washed with water $(2 \times 10 \mathrm{ml})$. Organic layer was dried with $\mathrm{MgSO}_{4}$, concentrated and passed through a plug of silica to yield $62.3 \mathrm{mg}(91.6 \%)$ of the trans ester 2 (0.03:1 cis:trans). 
2-[2-(2,6-Dimethoxy-4-pentyl-phenyl)-4-methyl-cyclohex-3-enyl]-2-propanol, 17<smiles>[CH2]c1cc(OC)c([C@H]2C=C(C)CC[C@H]2C(C)(C)O)c(OC)c1</smiles>

To the cyclized trans ester $2(0.546 \mathrm{~g}, 1.51 \mathrm{mmol})$ in $20 \mathrm{ml}$ ether at $-78^{\mathrm{O}} \mathrm{C}$ is added $\mathrm{MeLi}$ ( $4.5 \mathrm{ml}, 1 \mathrm{M}$ in ether, $4.5 \mathrm{mmol}$ ) drop wise with stirring. The cooling bath is removed and the reaction allowed to warm up to rt. When no starting material is visible by TLC the reaction is quenched with $10 \mathrm{ml}$ sat. $\mathrm{NH}_{4} \mathrm{Cl}$ solution, and the two layers separated. The aqueous layer is extracted with $(2 \times 20 \mathrm{ml})$ ether, the combined organic layers dried over $\mathrm{MgSO}_{4}$ and concentrated. The residue is columned with 7-10\% ethyl acetate- petroleum ether to yield $0.512 \mathrm{~g}(93 \%)$ of the desired alcohol. $[\alpha]^{\mathrm{D}}=-131.534\left(\mathrm{c}=0.86, \mathrm{CH}_{2} \mathrm{Cl}_{2}\right)$. IR (thin film): 3566.0, 3465.7, 2957.8, 2857.9, 1607.4, 1578.6, 1454.6, 1419.3, 1366.8, 1228.7, 1115.9, 976.1, 825.3 $\mathrm{cm}^{-1} .{ }^{1} \mathrm{H}$ NMR (500 MHz, $\left.\mathrm{CDCl}_{3}\right) \delta: 6.391(\mathrm{~s}, 2 \mathrm{H}), 4.993(\mathrm{~s}, \mathrm{br}, 1 \mathrm{H}), 3.96(\mathrm{~d}$, br, $J=9.644 \mathrm{~Hz}, 1 \mathrm{H}), 3.774$ (s, br, 6H), 2.528 (t, $J=7.935 \mathrm{~Hz}, 2 \mathrm{H}), 2.34-2.15$ (m, 2H), 1.9$1.96(\mathrm{~m}, 2 \mathrm{H}), 1.857(\mathrm{~s}, 1 \mathrm{H}), 1.644(\mathrm{~s}, 3 \mathrm{H}), 1.55-1.63(\mathrm{~m}, 2 \mathrm{H}), 1.28-1.38(\mathrm{~m}, 4 \mathrm{H}), 1.111(\mathrm{~s}$, $3 \mathrm{H}), 1.099(\mathrm{~s}, 3 \mathrm{H}) 0.896(\mathrm{t}, J=6.958 \mathrm{~Hz}, 3 \mathrm{H}) .{ }^{13} \mathrm{C} \mathrm{NMR}\left(125 \mathrm{MHz}, \mathrm{CDCl}_{3}\right) \delta: 142.70$, $132.05,125.29,119.86,74.22,55.84,46.88,36.42,33.4,31.63,31.07,30.57,28.4,26.42$, 25.75, 23.2, 22.54, 14.04. Elemental analysis calculated for $\mathrm{C}_{23} \mathrm{H}_{36} \mathrm{O}_{3} \mathrm{C}, 76.62 ; \mathrm{H}, 10.06$; found: C, 76.53; H, 9.98.

2-[6-(1-Hydroxy-1-methyl-ethyl)-3-methyl-cyclohex-2-enyl]-3-methoxy-5-pentyl-phenol, ${ }^{[3]}$ 18

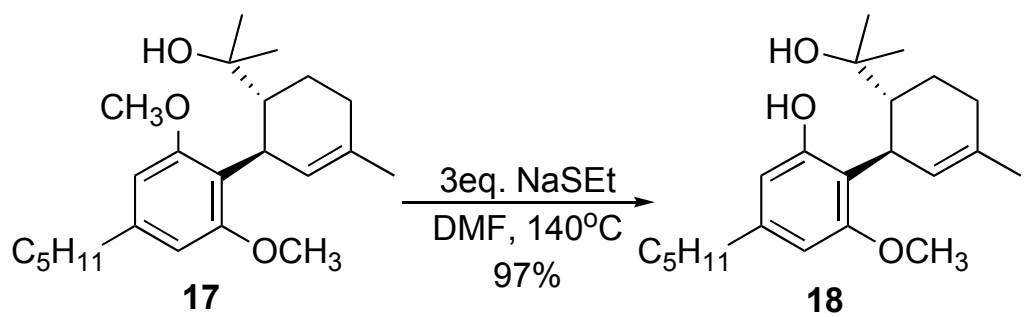

To the alcohol $(0.18 \mathrm{~g}, 0.499 \mathrm{mmol})$ in $10 \mathrm{ml}$ DMF was added NaSEt $(0.157 \mathrm{~g}, 80 \%$ content, $1.49 \mathrm{mmol}$ ) and the resulting heated at $140^{\circ} \mathrm{C}$ for $3 \mathrm{hr}$ till TLC showed no more starting material. The reaction mixture was cooled to room temperature, poured into $30 \mathrm{ml}$ aq. sat. $\mathrm{NaHCO}_{3}$ and extracted with ether $(3 \times 50 \mathrm{ml})$. The combined organic layers were dried over $\mathrm{MgSO}_{4}$, concentrated and the residue columned with $15 \%$ ethyl acetate- petroleum ether to yield $0.168 \mathrm{~g}(97 \%)$ of the desired phenol. 
$[\alpha]_{\mathrm{D}}^{23}-52\left(\mathrm{c} 0.79, \mathrm{CHCl}_{3}\right)$, lit. $[\alpha]_{\mathrm{D}}^{26}-44\left(\mathrm{c} 0.20, \mathrm{CHCl}_{3}\right){ }^{1} \mathrm{H} \mathrm{NMR}\left(500 \mathrm{MHz}, \mathrm{CDCl}_{3}\right) \delta$ : $6.34(\mathrm{~s}, 1 \mathrm{H}), 6.29(\mathrm{~s}, 1 \mathrm{H}), 6.06(\mathrm{~s}, 1 \mathrm{H}), 5.41(\mathrm{~s}, 1 \mathrm{H}), 4.034(\mathrm{~s}, 1 \mathrm{H}), 3.82(\mathrm{~s}, 3 \mathrm{H}), 2.50(\mathrm{t}, J=$ $7.935 \mathrm{~Hz}, 2 \mathrm{H}), 1.90-2.29(\mathrm{~m}, 5 \mathrm{H}), 1.754(\mathrm{~s}, 3 \mathrm{H}), 1.48-1.62(\mathrm{~m}, 3 \mathrm{H}), 1.24-1.38(\mathrm{~m}, 4 \mathrm{H}), 1.14$ $(\mathrm{s}, 3 \mathrm{H}), 1.12(\mathrm{~s}, 3 \mathrm{H}), 0.887(\mathrm{t}, J=6.958 \mathrm{~Hz}, 3 \mathrm{H}) .{ }^{13} \mathrm{C} \mathrm{NMR}\left(125 \mathrm{MHz}, \mathrm{CDCl}_{3}\right) \delta: 156.24$, $155.85,143.35,138.94,124.81,116.19,110.66,103.46,73.62$, 55.66, 47.9, 35.94, 32.95, $31.51,30.8,30.1,28.64,26.02,25.1,23.31,22.48,13.99$.

(-)-trans $-\Delta^{9}$-Tetrahydrocannabinol, ${ }^{[4]} \mathbf{1}$

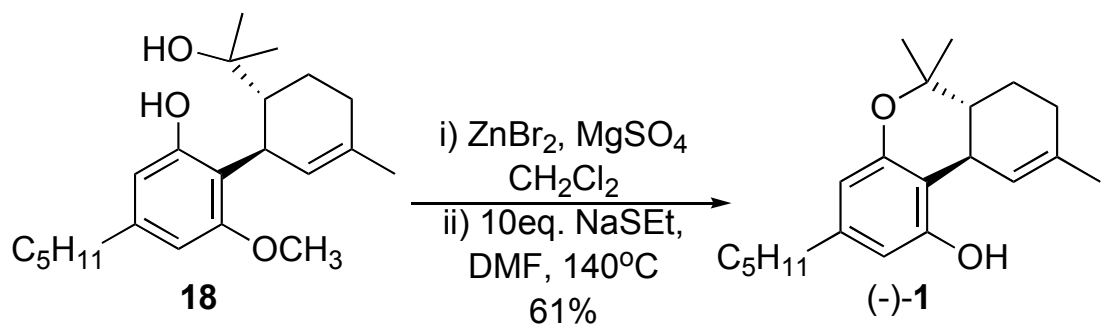

To a solution of phenol (82 mg, $0.2366 \mathrm{mmol})$ in $\mathrm{CH}_{2} \mathrm{Cl}_{2}(10 \mathrm{ml})$ were added $\mathrm{ZnBr}_{2}(106 \mathrm{mg}$, $0.473 \mathrm{mmol})$ and $\mathrm{MgSO}_{4}(200 \mathrm{mg})$ and the mixture stirred at room temperature overnight. It was then poured into sat. aq. $\mathrm{NaHCO}_{3}(30 \mathrm{ml})$ and extracted with ether $(2 \times 50 \mathrm{ml})$. The combined organic layers were dried over $\mathrm{MgSO}_{4}$, concentrated invacuo and the residue taken up in DMF (10ml). To this was added NaSEt $(250 \mathrm{mg}, 80 \%$ content, $2.36 \mathrm{mmol})$ and the resulting heated at $140^{\circ} \mathrm{C}$ for $10 \mathrm{hr}$. The mixture was then cooled to room temperature, poured into $30 \mathrm{ml}$ aq. sat. $\mathrm{NaHCO}_{3}$ and extracted with ether $(3 \times 50 \mathrm{ml})$. The combined organic layers were dried over $\mathrm{MgSO}_{4}$, concentrated and the residue columned with $10 \%$ ethyl acetate- petroleum ether to yield $45.5 \mathrm{mg}(61 \%)$ of the title compound.

$[\alpha]^{25}{ }_{D}-152\left(\mathrm{c} 0.79, \mathrm{CHCl}_{3}\right)$, lit. $[\alpha]_{\mathrm{D}}^{28}-150\left(\mathrm{c} 1.0, \mathrm{CHCl}_{3}\right){ }^{1} \mathrm{H} \mathrm{NMR}\left(500 \mathrm{MHz}, \mathrm{CDCl}_{3}\right) \delta$ : 6.297 (t, $J=1.586 \mathrm{~Hz}, 1 \mathrm{H}), 6.279$ (d, $J=1.465 \mathrm{~Hz}, 1 \mathrm{H}), 6.138(\mathrm{~d}, J=1.587 \mathrm{~Hz}, 1 \mathrm{H}), 4.708$ $(\mathrm{s}, 1 \mathrm{H}), 3.197(\mathrm{~d}, J=11.109 \mathrm{~Hz}, 1 \mathrm{H}), 2.38-2.47(\mathrm{~m}, 2 \mathrm{H}), 1.2-2.2(\mathrm{~m}, 8 \mathrm{H}), 1.68(\mathrm{~s}, 3 \mathrm{H}), 1.41$ $(\mathrm{s}, 3 \mathrm{H}), 1.091(\mathrm{~s}, 3 \mathrm{H}), 0.876(\mathrm{t}, J=6.958 \mathrm{~Hz}, 3 \mathrm{H}) .{ }^{13} \mathrm{C} \mathrm{NMR}\left(125 \mathrm{MHz}, \mathrm{CDCl}_{3}\right) \delta: 154.75$, $154.1,142.82$, 134.45, 123.65, 110.09, 108.99, 107.5, 65.88, 45.76, 35.46, 33.53, 31.49, $31.14,30.65,27.56,24.99,23.38,22.54,19.26,14.02$.

[1] Moore, M.; Rickards, R.W.; Ronneberg, H. Aust. J. Chem. 1984, 37, 2339. b) Mirrington, R.N.; Feutrill, G.I. Org. Synth. 1973, 53, 90.

[2] Adams; C. J. Amer. Chem. Soc. 1943, 65, 360, 361;Simon, J.W.; Holmes, A.M.; Johnson, I. Can. J. Chem. 1982, 60, 308; Achenbach, H. et al.; Chem. Ber. 1979, 112, 1841.

[3] William, A.D.; Kobayashi, Y. J. Org. Chem. 2002, 67, 8771. 
[4] Evans, D.A.; Barnes, D.M.; Johnson, J.S.; Lectka, T.; Matt, P.V.; Miller, S.J.; Murry, J.A.; Norcross, R.D.; Shaughnessy, E.A.; Campos, K.R. J. Am. Chem. Soc. 1999, 121, 7582. 